\title{
Deporte e identidad, o sobre cómo definirnos
}

\author{
Francisco Javier CASPISTEGUI \\ Universidad de Navarra \\ fjcaspis@unav.es
}

Recibido: 20 de febrero de 2012.

Aceptado: 10 de abril de 2012.

\begin{abstract}
Resumen
Este artículo trata de mostrar, a través de diversos ejemplos, la importancia del deporte en la configuración de los niveles identitarios en los que se han movido los seres humanos desde el siglo XIX. La asociación con el nacionalismo, regímenes totalitarios y dictaduras, no ha desgastado la capacidad de los deportes para vincular a las comunidades. En muchas ocasiones, esa capacidad integradora tiene un importante carácter defensivo, lo que impide considerar las prácticas deportivas como mero reflejo de la modernidad. De ahí la necesidad de buscar la historización de las identidades deportivas, contextualizando y buscando el análisis interdisciplinar para evitar visiones reduccionistas.
\end{abstract}

Palabras clave: Deporte; identidad; modernidad defensiva; nación.

\section{Sport and identity, or how to define ourselves}

\begin{abstract}
This article tries to show through various examples, the significance of sport in the shaping of human beings' identities since the nineteenth century. The association with nationalism, totalitarian regimes and dictatorships, has not eroded the ability of sport to connect communities. In many cases, this inclusiveness has an important defensive feeling, preventing to consider sports practices as a mere reflection of modernity. Hence the need to historicize sporting identities, contextualizing and seeking interdisciplinary analysis to avoid reductionist views.
\end{abstract}

Key words: Sport; identity; defensive modernity; nation.

\section{Referencia normalizada}

Caspistegui, F. J. (2012). Deporte e identidad, o sobre cómo definirnos. Historia y Comunicación Social, Vol. 17, páginas 19-39.

Sumario: 1. Introducción. 2. ¿Deporte moderno? 3. Deporte moderno e identidad. 4. Conclusiones. 5. Bibliografía.

\section{Introducción}

El 21 de noviembre de 1920, tropas británicas abrieron fuego sobre jugadores y asistentes al partido de fútbol gaélico entre el Dublín y el Tipperary. Murieron 14 personas. Respondían al asesinato de 14 oficiales de inteligencia británicos la noche previa. El marco fue el estadio Croke Park de Dublín, sede de la Gaelic Athletic Association (GAA), quintaesencia deportiva irlandesa a través de los juegos gaélicos (Mandle, 1987; Burca, 1999; Cronin, 1999; Mcanallen, Hassan, Hegarty, 2009; 
Cronin, Murphy, Rose, 2009. Sugden, Bairner, 1993; Bairner, 2005). Esta conexión identidad irlandesa-deporte adquirió un acusado significado simbólico con la visita que Isabel II realizó a Irlanda en mayo de 2011 (Burke-Kennedy, 18.05.2011; Bloxham, 18.05.2011; The New York Times, 18.05.2011). El segundo día del recorrido acudió al estadio donde se reunían dos elementos de especial significado, histórico uno, tradicional el otro ${ }^{1}$, en un gesto que buscaba superar los recelos entre ambos países. En ese acercamiento simbólico, la reina saludó a varios jugadores, incluidos uno del Dublín y dos del Tipperary. Pero aunque la Asociación había aceptado la visita y sus integrantes recibieron una carta recomendando evitar polémicas, algunos se negaron a estar presentes en el que consideraban un monumento de la "guerra de la independencia"2. Además, el estadio permaneció vacío, privando de una parte del significado reconciliador que se pretendía lograr. Pese a ello, el presidente de la GAA, Christy Cooney, resaltó ante la reina británica el profundo carácter irlandés de la asociación, que "has consistently embodied the mood of the nation, culturally, socially and politically". Hizo referencia a las turbulentas relaciones anglo-irlandesas, a la necesidad de no olvidar a quienes perdieron su vida, pero a su vez, al impulso que como Asociación habían dado al proceso de paz, que consideró irreversible ${ }^{3}$.

Pasado y presente, identidad nacional e histórica, símbolos y mitos en una mezcla que pese a las dificultades, acabó funcionando. La pregunta podría ser si el deporte ha actuado como elemento cohesivo o si fue la política la que absorbió el papel de mediación que aquél proporcionó. Sea cual fuere la respuesta, resulta evidente que uno de los actos más simbólicos de la (también) histórica visita, tuvo como epicentro un marco deportivo. ¿Es el deporte la -o una-canalización de las identidades nacionales? ¿Cuál es su papel en la configuración de las comunidades, sean del tamaño que sean? ¿Debemos, desde las ciencias sociales, prestar atención al papel del deporte como elemento aglutinador $-\mathrm{y}$ rupturista-? ¿Se canaliza con más facilidad el sentimiento de patria o de nación a través del ritual deportivo? ¿Es más sencilla la integración en una identidad consolidada y estable a través del deporte? ¿Facilita la creación de identidades grupales? La respuesta a buena parte de estas preguntas puede parecer de sentido común. Sin embargo, la trayectoria histórica de las prácticas deportivas alcanza ya un espesor temporal y geográfico considerable, por lo que la variabilidad de los casos obliga a precaverse ante generalizaciones apresuradas. Algunos análisis adscribieron las prácticas deportivas al impulso y consolidación de la burguesía y al establecimiento por ésta de pautas de organización y auto-percepción que la definían. Uno de los mecanismos para ello fueron los deportes, especialmente los considerados más adecuados a los ideales sociales defendidos, con una especial insistencia en su carácter aficionado y en la capacidad para vincular en organizaciones estables a sus integrantes (Hobsbawm, 1993: 141; Walton, 2001). Pudiera decirse que los deportes se destinaban a la protección de los grupos sociales más favorecidos con normas y reglas frente a las prácticas, generalmente violentas, de las clases populares ${ }^{4}$. Los deportes sometidos a control, amateurs, mostraban una faceta más de la modernidad triunfante que las pujantes clases medias protagonizaron, con evidente exclusión de otros grupos. 
Sin embargo, las transformaciones que en el siglo XIX sacudieron las sociedades occidentales, implicaron una rápida generalización de los deportes, bien para canalizar las aspiraciones de los no burgueses, bien para someter a otras formas de control unas prácticas crecientemente comercializadas ${ }^{5}$. En este marco se mostró la potencialidad integradora y la capacidad para generar emociones que el deporte implicaba, algo que se hizo más perceptible conforme el nacionalismo se consolidaba como la gran fuerza política e ideológica desde fines del siglo XIX. Dado que el deporte tendía a incorporar adeptos de sectores sociales cada vez más amplios; dado su interés económico a través de los espectáculos de masas; dada su creciente importancia en las relaciones entre naciones y grupos sociales, su utilidad política, ideológica e identitaria fue una manera de defender los esquemas sobre los cuales se había construido el entramado social. Este proceso alcanzó un especial desarrollo en el siglo XX, cuando el deporte se asumió como tarea estatal, tanto en sentido político como social ${ }^{6}$. De forma paralela, la creación de organizaciones deportivas supra-nacionales implicó la necesidad de someter muchas de las prácticas locales al marco más amplio, global, que el deporte estaba alcanzando, desde el punto de vista normativo, de su difusión e interés económico.

Esta evolución se comenzó a analizar tanto por la sociología del deporte ${ }^{7}$, como, más tardíamente, por la historia ${ }^{8}$. Esta última ha tendido a incluir todo el proceso en el marco de dos etapas históricas: pre-moderna y moderna ${ }^{9}$. Esta clasificación, útil desde un punto de vista interpretativo, ha recibido críticas por su esquematismo y por ignorar las resistencias y continuidades a ese modelo, muy acorde con una explicación histórica apoyada en la idea de progreso. Podría añadirse una crítica más, el olvido de elementos como pertenencias, identidades y conexiones nacionales-locales del deporte. No hay que ignorar que a comienzos del siglo XXI bien puede hablarse de deporte global en algunos aspectos (económico y organizativo), pero también de deporte local por la pervivencia de sentimientos localistas-nacionalistas (lo condensado en el término glocal). Frente a las reflexiones que consideraban superado el nacionalismo como fuerza significativa, éste muestra su pervivencia en algunos elementos de nuestra época, y especialmente en el deporte. Cuando en 1995 Michael Billig publicó Banal nationalism, buscaba recoger la cotidianeidad de signos, comportamientos, actitudes y simbología más allá de las grandes manifestaciones de fe nacionalista. Trataba de demostrar que no son necesarios despliegues mediáticos, ni campañas institucionales para convencer a amplios grupos de población, puesto que con un simbolismo difuso, con la reiteración de comportamientos y actitudes, podría obtenerse o mantenerse un patriotismo fácil de movilizar en circunstancias especiales (Billig, 1995). En último término, la identidad nacional sería "more than an inner psychological state or an individual self-definition: it is a form of life, which is daily lived in the world of nation-states" (Billig, 1995: 69). En ello influía el nombre de la nación, su historia y geografía, la unidad territorial imaginada, los estereotipos, la inserción internacional a través de la bandera y el himno, las prácticas discursivas, el lenguaje deíctico, los mapas del tiempo..., y también el deporte, omnipresente en todos los medios de comunicación. Detrás de ello asoman las ideas de Benedict Anderson o Eric Hobsbawm sobre las naciones como "comunidades 
imaginadas", grupos humanos vinculados no por un imposible contacto, sino por un conjunto de convenciones e instrumentos (las tradiciones inventadas de las que habla el segundo) que crean la ilusión del grupo homogéneo (Anderson, 1993; Hobsbawm, Ranger, 1996). Aunque estas explicaciones dejan de lado la efectividad práctica de los sentimientos colectivos generados ${ }^{10}$, muestran hasta qué punto es posible construir elementos que favorezcan una determinada identidad. Desde fines del siglo XIX y sobre todo en el siglo XX, el deporte ha sido tal vez uno de los instrumentos más utilizados en la consecución de esos lazos, en la invención de esas comunidades, donde se repiten "the commonplace stereotypes of nation, place and race, not to mention those of masculinity [...] sharing in defeats and victories, feeling at home in this world of waved flags" 11 . De hecho, las conexiones entre el deporte y la guerra - dos de los principales elementos nacionalizadores- han sido habituales en esos medios, indicando tanto la preparación para el combate, como una vía para dirimir supremacías de forma incruenta:

Day after day, millions of men seek their pleasures on these pages, admiring heroism in the national cause, enjoying prose which intertextually echoes warfare. Such pleasures cannot be innocent. If nationhood is being flagged, then the routine reminders might also be rehearsals; the echoes of the past cannot be discounted as preparations for future time (Billig, 1995: 125; Mignon, 1999).

\section{2. ¿Deporte moderno?}

Un hito en este proceso de apropiación del deporte como instrumento identitario fueron los Juegos Olímpicos. Cuando el barón de Coubertin buscó recuperar la esencia de los viejos juegos griegos, no trataba de rescatar una referencia histórica. Las prácticas deportivas regladas, la distinción social que proporcionaban como síntoma de estatus y modernidad fueron aspectos que paulatinamente quedaron superados. Y ello se debió en primer lugar a la extensión de estas prácticas. A fines del siglo XIX se democratizaron los entretenimientos públicos al amparo del creciente tiempo de ocio que la organización socio-económica permitía, especialmente en el mundo urbano occidental. Cada vez más sectores sociales se planteaban la práctica -emulando comportamientos caballerescos como el fair play o deportivismo-, y -aún más- la asistencia a espectáculos deportivos. En segundo lugar a la percepción de su utilidad como fuente de robustez no ya individual, sino colectiva, muy vinculada a quienes habían impulsado la puesta en práctica de los deportes: los ejércitos. La fortaleza física se convirtió en indicador del prestigio nacional. Y, en tercer lugar, como una reivindicación de la masculinidad, vinculada al componente militar y a la extensión del tiempo de ocio entre los trabajadores (Dunning, 1994), con la participación laboral femenina restringida al hogar, de nuevo emulando los patrones sociales dominantes.

Los Juegos Olímpicos, frente a ello, surgieron como cierta forma de defensa frente a un mundo en radical transformación. Y aunque el deporte era síntoma de modernidad, se empleó frente a la amenazante percepción de esa modernidad. Así, el 
elitismo de clase media y su extensión más allá de sus orígenes británicos, se refugiaba en un ideal deportivo aficionado, caballeresco y vinculado al estado-nación. Esta actitud restringía la participación de una gran mayoría de los grupos sociales que accedían masivamente al deporte, sobre todo desde el profesionalismo (Hoberman, 1995). La democratización cultural no acababa de resultar grata y se reaccionaba mediante el esnobismo, la creación de reductos blindados frente a esas aspiraciones. Por otro lado, y aunque los estados asumieron la extensión social del deporte, patrocinaron la participación nacional en los acontecimientos deportivos más elitistas, lo que reforzó las relaciones con el poder de esas élites, convirtiendo a los Juegos Olímpicos en una gran plataforma de confrontación nacional, donde los estados ponían en juego su prestigio, se mostraban a sí mismos y a la nación que encabezaban con unos rasgos que pregonaban la esencia de su identidad, la conciencia de su diferencia (Mangan, 1985; Milza, 2004). Por último, redujeron el ámbito deportivo a una muestra de masculinidad, ya establecida en lo laboral y tradicionalmente arraigada en la milicia. No en vano los Juegos Olímpicos no admitieron mujeres en el atletismo hasta 1928 (Mitchell, 1977).

Ante el cambio acelerado y las transformaciones se buscó el control del deporte, y además se logró una aculturación desde arriba que encontró eco en el conjunto de la sociedad. Sin embargo, esta expansión y adaptación no implicaba necesariamente una aceptación acrítica, y pronto surgieron respuestas frente a la homogeneización. Por un lado estaban aquéllas que rechazaban los modelos inspirados en Coubertin, como la creación de circuitos deportivos obreros (Arnaud, 1994; Kruger, Riordan, 1996. Jones, 1988; Holt, 1990. Wheeler, 1978; Naison, 1979), así como la puesta en práctica de Olimpiadas Obreras, paralelas a las impulsadas por el COI (Steinberg, 1978; Jones, 1988: 164-95; Riordan, 1991; Baker, 1992; Mcquarrie, 2010), que mostraron el repudio hacia ese modelo basado en las marcas y en la competitividad exacerbada. Por otro lado, la permanencia de prácticas "pre-modernas" adaptadas a los nuevos modos sociales y convertidas en refugio de identidades locales, regionales o nacionales frente a la modernidad de los deportes importados. Por último la creciente profesionalización, un modelo alternativo de deporte disociado de las prácticas amateurs, que se vinculó al peso social de los espectáculos, y que creció de forma exponencial gracias en parte a su fuerza identitaria.

De todo ello surge una primera reflexión, y es que el deporte sirvió para articular formas diversas de comprensión de la identidad. Alcanzaba con su influencia todos los estratos sociales, al estado, la creciente sociedad civil y se afirmaba en el espacio público. Mostraba eficacia y utilidad, capacidad de adaptación y penetraba en todos los ámbitos, con la salvedad renuente de los intelectuales, consolidados como grupo a la vez que el deporte. En segundo lugar, señalar que el deporte combinaba en sus primeros pasos la modernidad, por su condición urbana, por el proceso civilizador que implicaba, por sus pretensiones democratizadoras, por impulsar el desarrollo personal y la preocupación hacia cada individuo y por proponer un tipo de relación entre estados más tolerante e integradora; preservaba modelos sociales de clase media, estables y de orden al modo occidental, con una masculinidad predominante, con la capacidad para aunar, favorecer relaciones y crear comunidad, y con un 
modelo de trato internacional apoyado en el estado-nación. Como indicábamos, esta dualidad paradójica (integrar/excluir, nacionalizar/internacionalizar, modernizar/ preservar) provocó reacciones que mostraban las grietas en su percepción. Sin embargo, no se cuestionó el deporte, pues su flexibilidad permitía una constante adaptación. Algunos ejemplos pueden ayudar a percibirlo.

Desde fines del siglo XIX se extendió la idea de que el deporte era un factor de modernidad, pues el esfuerzo aplicado a su práctica reflejaba la capacidad de quien lo ejecutaba para la mejora, para el progreso, de los que el deporte se convirtió en un significativo proveedor de símbolos, asumidos por las autoridades. En EE.UU. se hablaba de la construcción de una república deportiva a partir de pautas griegas, lo que ayudaba a vincular prácticas físicas y modelo político. La Grecia clásica podía ofrecer un antídoto frente a los excesos modernizadores, permitía usar la referencia unificadora que los juegos habían tenido para aplicarla al presente de un país diverso y complejo (Dyreson, 2008: 189; Pope, 1997; Gems, 2006. Kyle, 1990). También en la Alemania de fines del XVIII y comienzos del XIX, el referente helénico proporcionó una alternativa al cosmopolitismo ilustrado, aportando argumentos para la consolidación de una identidad germánica. El panhelenismo de los juegos olímpicos se aplicaba a una compleja fragmentación política, pero cohesionada por lazos culturales y lingüísticos. Valoraban el modelo griego de los juegos y la importancia identitaria del deporte como factor de refuerzo de la nación cultural, más allá de la existencia de un estado-nación. El deporte sirvió como instrumento para potenciar la vitalidad de los alemanes frente a los ejércitos ocupantes. El paso hacia el nacionalismo político lo asumió Friedrich Ludwig Jahn y fue la base para una percepción nacionalista del deporte que triunfó en otros países (Saure, 2009).

Ambos casos abusan del modelo griego, pero más allá de la exactitud en su empleo, interesa su utilidad inmediata, como medio de difusión de los valores, de la cultura política y de la identidad de dos estados-nación en formación. No en vano, las primeras participaciones de EE.UU. en los Juegos Olímpicos buscaron el triunfo global y así lo proclamaron, pese a que no fue real. Pero era su forma de mostrar simbólicamente que mediante el deporte, la joven nación que representaban superaba las debilidades europeas y mostraba la excepcionalidad de lo que algunos autores consideran el triunfo de una hegemonía cultural ${ }^{12}$. Esta forma estadounidense de interpretar las Olimpiadas se generalizó desde fines de los años veinte y el objetivo fue, cada vez más, la victoria. Triunfaba un nacionalismo atlético (Macaloon, 1984: 241-80).

El caso de China es similar en la primera mitad del XX (Hong, Hua, 2002; Morris, 2004. Jarvie, Hwang, Brennan, 2008; Zhouxiang, Hong, 2010; Zhouxiang, 2011), pues medio siglo antes era ya un instrumento mediante el cual hacer frente a las amenazas exteriores. Buscaban superar la imagen del "hombre débil de Asia", e importaron modelos y tutela deportiva occidental -alemana y norteamericana-; se incorporó la idea de la vigorización para hacer frente a la debilidad física y moral, y se buscó con ahínco la participación en competiciones que mostraran los resultados: a nivel interno, desde 1910; en encuentros internacionales, en los Campeonatos de Asia desde 1913 -Shanghai, 1915- (Abe, 2007), y con especial insistencia en las 
citas olímpicas. La primera participación, con un atleta, tuvo lugar en 1932. La finalidad no era tanto deportiva como política y nacionalista: mostrar al mundo el rechazo a la pretensión japonesa de enviar representantes del estado títere de Manchuria. La delegación en Berlín de 1936 alcanzó los 69 atletas. Estas competiciones entre naciones servían como indicador del éxito nacional, reflejo de la capacidad para alcanzar la modernidad y como una forma de legitimación, teniendo en cuenta que China era republicana desde 1912, y las amenazas japonesas. A su vez, servían como eficaz instrumento para generar conciencia nacional (Morris, 1999; Dongguang, 2002; Hong, Mackay, Cristensen, 2008; Guoqi, 2008).

Sin embargo, estas visiones unívocas no ocultaban tensiones. China luchó contra la presencia occidental mediante el impulso de las artes marciales, acordes con la perspectiva nacionalista, con la finalidad militarista y defensiva, y con la reivindicación de su papel internacional ${ }^{13}$. En EE.UU se apreciaba a través del boxeo profesional, una suerte de heterodoxia de acuerdo a los patrones atléticos consolidados. Así lo manifestaron simbólicamente los combates entre Jack Dempsey y Gene Tunney de 1926 y 1927. El primero, de origen marginal, mostraba un grupo social muy distinto al encarnado por el segundo, cuyas maneras y trayectoria lo asimilaban al ideal de clase media. La victoria de Dempsey ofreció un modelo compensatorio, pues frente a las exigencias crecientes de una sociedad compleja, su trayectoria, como la de otros héroes populares, permitía la esperanza de acceder al éxito por vías menos ortodoxas ${ }^{14}$. Se trataba más de una negociación de identidades que de un conflicto. No surgieron universos paralelos en lo deportivo, se tendió a buscar lo común, a integrar al "otro" dentro de la "ortodoxia" social, de la cultura hegemónica, comenzando por los inmigrantes. Jesse Owens, Joe Louis, L.F. Sockalexis y G.H. Johnson, son ejemplos de héroes deportivos que no plantearon críticas al modelo segregacionista y que reforzaron la imagen integradora de EE.UU. ${ }^{15}$ Lo mismo podría decirse de asociaciones deportivas de carácter étnico, como la Mexican Athletic Association of Southern California, enlazada con México, pero en busca de su integración en EE.UU.; o los jugadores del Buffalo Germans de baloncesto, seguidores del Turnen, pero a su vez, participando de forma activa en su entorno ${ }^{16}$. Tampoco la inicial participación femenina en el deporte supuso una amenaza a los modelos vigentes, y ello pese a que desde muchos sectores sí se consideró su mera presencia como un serio desafío al predominio masculino (Guttmann, 1991; Arnaud, Terret, 1996; Caspistegui, 2004).

\section{Deporte "Moderno" e Identidad}

Después de 1945, los vínculos entre deporte e identidad se reforzaron a través de los estados nacionales (Polley, 1998: 12-62; Smith, Porter, 2004). El uso masivo que los derrotados habían hecho de las prácticas deportivas no supuso su rechazo, al reforzar los valores éticos que encerraban, una forma de responder a los abusos totalitarios, al racialismo y al afán de superioridad. El uso totalitario del deporte no cerró las puertas a su empleo -en parte por su utilidad identitaria-, a que se convirtiera 
incluso en plataforma de resistencia y, al menos, en una vía para afirmar pertenencias e identidades heterodoxas ${ }^{17}$. Así ocurrió, por ejemplo, en la Italia fascista:

While the regime promoted its ideal of an organic, patriotic, nationalist and united nation through football, the reality was often very different. Although calcio was an effective vehicle for promoting and disseminating the idealized Fascist, national community, occasionally it also drew considerable attention to the strong regional identities that existed throughout the peninsula (Martin, 2004: 3. Véanse: Fabrizio, 1976; Arnaud, Riordan, 1998; González Aja, 2002).

El fútbol aportó una base para mostrar cierta disconformidad en la URSS. Desde su fundación en 1935, el Spartak de Moscú supuso una pequeña forma de decir no al régimen soviético, en parte por no estar vinculado a organismos del régimen, como el Dínamo (a la policía secreta) o el CSKA (al ejército). La elección podía indicar -e indicaba- más que una mera opción deportiva pues, para empezar, era uno de los pocos ámbitos en los que se podía optar sin constricciones. Además, constituía una forma inocua de situarse frente a las estructuras del régimen $-y$ gritar en el estadio "muera la policía"-. Estaba su denominación cargada de simbolismo, como rebelión de los más explotados frente a la opresión. Pronto fue conocido como el equipo del pueblo, lo que se reforzó tras sus victorias de 1938 y 1939. Esto no implicaba, en modo alguno, la creación de identidades anti-soviéticas, ni siquiera de un "nosotros" frente a un "ellos", sino más bien aprovechar islas de autonomía ${ }^{18}$. De igual modo, la adopción del baloncesto como deporte nacional en Lituania a partir de jugadores y entrenadores norteamericanos de origen lituano, tuvo mucho que ver con los éxitos de este país en las competiciones internacionales, como los campeonatos europeos de 1937 y 1939. Quedó como el recuerdo de lo que había sido como nación y de lo que podía recuperar (Senn, 1988; Cingiene, Laskiene, 2004; Carlson, 2011).

En cualquier caso, los rasgos del deporte y su relación con las identidades siguieron siendo muy similares a los de los momentos iniciales. Tanto la capacidad de integración e identificación que proporcionaba, como la paradójica modernidad defensiva que en muchas ocasiones manifestaba, se mantuvieron en las décadas siguientes a la guerra. El deporte se asumía ya como tarea fundamental de los estados, no en sentido totalitario, sino en la recomendación para la práctica y en la disposición de recursos para ésta. Esto reforzaba el carácter moderno del deporte, asociado a la creación de sociedades de bienestar y a la capacidad de integración y convivencia entre comunidades ${ }^{19}$. Los niveles de práctica deportiva se consideraban indicadores fiables del grado de progreso de una sociedad. Su papel como instrumento de relación entre estados ya no asumía los denigrados modelos militaristas, sino más bien un componente pacifista, una extensión de las prácticas del fair play a la convivencia internacional. Por último, este tiempo ha mostrado el auge del deporte espectáculo, lo que ha seguido siendo utilizado como instrumento de cohesión o reivindicación nacional, además de implicar su inserción en las tendencias globalizadoras, con un deporte universalizado en sus grandes acontecimientos, con el intercambio frecuente de jugadores y con una abrumadora lógica económica.

Pero igualmente, las paradojas siguieron siendo un rasgo ineludible. Frente a la 
universalización se defendió la particularidad deportiva y la nacionalización de prácticas específicas, constituidas como elementos identificativos y simbólicos. Frente a quienes argüían que el nacionalismo estaba en trance de desaparición, el deporte se ha convertido en uno de sus refugios -o plataformas- (Houlihan, 2000: 222), merced en parte a su estrecha alianza con los medios de comunicación y las innovaciones técnicas que éstos han aportado (Hand, Croley, 2005; Blain, Boyle, O'Donnell, 1993; Gebauer, 1994. Baker, Boyd, 1997); en ocasiones se combinó la faceta más universalista e integradora con manifiestas defensas de una propuesta nacional -como en las ceremonias de apertura de los Juegos Olímpicos- o incluso como respaldo a regímenes dictatoriales (Archetti, 2004; Lee, Bairner, 2009); pero también con encendidas reivindicaciones de unidades sub-estatales, por no mencionar la reafirmación de identidades regionales, locales, etc. Por su parte, y a nivel internacional, más allá de los discursos de buena voluntad, el deporte se convirtió en instrumento de política internacional, con sus principales organismos (COI, FIFA) como actores cada vez más influyentes en el teatro diplomático. Así pudo apreciarse sobre todo con la incorporación de la URSS a las competiciones internacionales, que añadió un nuevo campo de juego durante la guerra fría, con diversas auto-exclusiones; o el veto a la participación de Sudáfrica por el mantenimiento del Apartheid. Veámoslo a través de algunos ejemplos.

El caso alemán es significativo. En los años cincuenta, su admisión en la UEFA (1954) se interpretó como la recuperación del estatus internacional perdido tras la guerra y junto a su victoria en el mundial de ese mismo año, celebrado en Suiza ("el milagro de Berna"), sirvieron como un refuerzo identitario, al asociarse el triunfo deportivo con la normalización externa y el prestigio internacional. Del mismo modo, tras el proceso unificador posterior a 1989, los equipos deportivos unificados buscaron encarnar a una nueva Alemania. Por una parte mostraba el triunfo del modelo occidental, pero a su vez, al incorporar deportistas de la extinta RDA, construía un modelo integrador, la recepción de los hijos pródigos: "Le sport confère au nationalisme una forme sensible. Et le nationalisme redonne un sens à un sport professionnel vidé de son contenu" (Gebauer, 1994: 107; Hesse-Lichtenberger, 2003: 119-32; Krauss, 2003; Heinrich, 2003; Jordan, 2005; Brüggemeier, 2010). Es significativo que obtuvieran el mundial de fútbol 1990.

El otro país excluido fue Japón. Sin embargo, a partir de los cincuenta, uno de sus intentos de transformación fue el uso del deporte como vía para la integración, el reconocimiento externo y la auto-identificación. Solicitó la celebración de unos juegos desde 1952, bien consciente su gobierno de la importancia simbólica de dicha petición. No los obtuvo para 1960, pero volvió a solicitarlos para 1964 y lo logró (Fasold, 2002). Una vez obtenido el primer objetivo, se plantearon el segundo en la misma línea: lograr el reconocimiento del judo -donde el débil puede vencer al fuerte-como disciplina olímpica con la finalidad última de obtener las medallas que reforzaran el mensaje de integración y prestigio internacional, además del valor del esfuerzo individual encarnado en los héroes surgidos de la práctica deportiva y como respaldo a una identidad que se trataba de regenerar sobre postulados tradicionales, incluso recuperando elementos propios del período pre-bélico ${ }^{20}$. 
El deporte parecía superar su asociación con el imperialismo cultural o con la ambición de dominio, aunque el éxito deportivo se buscaba como muestra de prestigio nacional y de cierta primacía moral ${ }^{21}$. La pregunta podría ser entonces si el deporte contribuía al refuerzo de una idea, la del nacionalismo, que a lo largo del siglo XX fue entrando en un aparente declive. ¿Sirvió el deporte como una reserva del sentimiento nacional, como uno de los depósitos en apariencia banales de ese nacionalismo en retroceso político e ideológico, pero muy activo en lo sentimental, en la identidad de fondo de muchos ciudadanos europeos? ¿Estamos ante una glocalización, es decir, la capacidad de resistencia que el deporte y el nacionalismo han mostrado frente a la globalización pero usando los instrumentos y mecanismos de ésta?22 Parece que la respuesta habría de ser afirmativa, pero también existirían paradojas, al mostrar un intenso orgullo nacional ante las selecciones nacionales, pero una escasa correspondencia con actitudes políticas:

En divisant clairement les intérêts économiques des clubs et l'autonomie des équipes nationales, les autorités du football ont réussi à faire coexister deux modèles antithétiques: les grandes multinationales régies par les lois du marché et un modèle d'équipes nationales relativement indépendant des contingences économiques. C'est grâce à cette dualité que perdure la passion pour les matches de football, mélange de l'essentiel et du dérisoire, aux quatre coins du globe (Lanfranchi, 2002: 24).

Un ejemplo de ello es el de James Sillars, del Scottish Nationalist Party, en las elecciones de 1992, para quien el patriotismo de Escocia se limitaba a noventa minutos, al constatar el mínimo respaldo a sus propuestas, aun cuando en un partido previo contra Inglaterra, la emoción alcanzó grados superlativos ${ }^{23}$. Quizá la respuesta haya de venir de la constante actualización de los conceptos y de la necesidad de hacer frente al análisis de estas cuestiones a partir de las circunstancias de su propio tiempo. Los comportamientos no se ajustan a modelos puros, sino que emplean unos y otros, en muchos casos de forma defensiva. Habría que tener en cuenta, además, la variabilidad de los nexos entre deporte, nación e identidad y en relación a la globalización, que distan mucho de ser inmutables, y más en tiempos como los presentes, de aceleración constante (Maguire, 1999 y 2006; Miller, 2001). Serviría también para tener en cuenta esos componentes emocionales, canalizados a través del ritual de los himnos, las banderas, la exaltación a través de los medios de comunicación, con un papel creciente en la afirmación de identidades: "sport and globalization have become accomplices in the process whereby the importance of national identity has been ensured" 24 . ¿Podremos entonces seguir hablando de la relación entre deporte y globalización o más bien, como se ha propuesto, entre deporte e internacionalismo o cosmopolitanismo? ${ }^{25}$

Tal vez ahí haya que introducir la propuesta de Giulianotti, al añadir al esquema dual de explicación histórica del deporte (pre-moderno/moderno), un tercer elemento, el posmoderno ${ }^{26}$. Trataría este nuevo espacio de comprender, por ejemplo, la presencia en las selecciones nacionales de deportistas no nacidos en el país al que van a defender; o el refuerzo de las identidades deportivas locales o regionales, en muchas ocasiones como forma de defensa frente a la globalización deportiva y la 
pérdida de referentes consiguiente a ésta, pero también la lógica económica que rige la práctica deportiva y las contradicciones que ésta muestra respecto a los modelos identitarios rígidos (Silk, Andrews, Cole, 2004; Amish, Cornwell, 2005).

\section{Conclusiones}

En definitiva, la cuestión de la identidad en el deporte está sujeta a la intensa variabilidad de los conceptos y a las situaciones en las que se aplica, lo cual no implica una relajación de los lazos entre ambas sino, más bien, la necesidad de prestar atención a la multiplicidad de los establecidos. Provistos de herramientas conceptuales e interpretativas rígidas o muy apegadas a disciplinas y orientaciones monocausales, corremos el riesgo de no comprender un elemento que muestra una imagen de modernidad y la ejemplifica en muchos aspectos, pero que, a su vez, es capaz de preservar algunos de los aspectos más característicos de un mundo que parece en vías de desaparición. De ahí la necesidad de considerar al deporte en su relación con el mundo que le rodea y, especialmente, con las filias y fobias que genera, como una modernidad defensiva, un instrumento destinado a proteger(se) de la aceleración a la que sometemos nuestra vida.

Acaso haya que pensar que cuando la reina Isabel II visitó Croke Park, defendía una identidad, la británica, frente a la historia encarnada en el "domingo sangriento"; defendía también, mediante el reconocimiento y el respeto, la identidad irlandesa y con ella, la posibilidad de una convivencia entre comunidades. Identidad irlandesa defendida, por último, a través de unos juegos gaélicos considerados como signos de la esencia nacional. Los simbolismos de la identidad jugaron a través del deporte un significativo papel, universalizando el encuentro entre ingleses e irlandeses mediante la afirmación de lo propio y distintivo. Nación, deporte e identidad no parecían mostrar síntomas de distanciamiento, aunque sus relaciones fluctuasen dependiendo de las circunstancias y de las miradas que sobre ellas vamos arrojando. En definitiva, la identidad nacional, regional, local, grupal... es una sensación de pertenencia colectiva, pero también la condición mediante la cual sobreviven los estados y las organizaciones apoyadas en cada una de esas identidades. Como construcciones de sentido y aunque estén fundamentadas sobre bases más o menos reales, las identidades grupales requieren ser alimentadas de forma constante y, por ello, constantemente repensadas. Y en ese proceso, parece indudable, el deporte juega un papel de suma importancia.

\section{Bibliografía}

ABBASSI, D. (2007). "Sport et usages politiques du passé dans la Tunisie des débuts du XXIe siécle". En: Politiques et Sociétés, 26/2-3. p. 125-42.

ABE, I. (2007). "Historical significance of the Far Eastern Championship Games: an international political arena". En NIEHAUS, A.; SEINSCH, M. (eds.) (2007). 
Olympic Japan. Ideals and realities of (inter)nationalism. Würzburg: Ergon Verlag. p. 67-88.

ABELL, J. et al. (2007). "Who ate all the pride? Patriotic sentiment and English national football support". En: Nations and Nationalism, 13/1. p. 97-116.

ALAMILLO, J.M. (2010). "Plating across the borders: transnational sports and identities in Southern California and Mexico, 1930-1945”. En: Pacific Historical Review, 79/3. p. 360-92.

AMISH, J.M.; CORNWELL, T.B. (2005). Global sport sponsorship. Oxford: Berg.

ANDERSON, B. (1993 [1983]). Comunidades imaginadas. Reflexiones sobre el origen y la difusión del nacionalismo. México: FCE.

ARCHETTI, E.P. (2001). El potrero, la pista y el ring. Las patrias del deporte argentino. México: FCE.

(2004). "El mundial de fútbol de 1978 en Argentina: victoria deportiva y derrota moral". En: Memoria y Civilización, 7. p. 175-94.

ARNAUD, P. (ed.) (1994). Les origines du sport ouvrier en Europe. París: L'Harmattan. RIORDAN, J. (eds.) (1998). Sport and international politics. The impact of fascism and communism on sport. Londres: E.\&F.N. Spon.

TERRET, T. (eds.) (1996). Histoire du sport féminin. París: L'Harmattan.

BAIRNER, A. (1996). "Sportive nationalism and nationalist politics: a comparative analysis of Scotland, the Republic of Ireland and Sweden". En: Journal of Sport and Social Issues, 20/3. p. 314-35.

(2001). Sport, nationalism and globalization: European, and North American perspectives. Nueva York: Suny Press.

(2005). Sport and the Irish: histories, identities, issues. Dublín: University College Dublin Press.

(2009). "National sports and national landscapes: in defence of primordialism". En: National Identities, 11/3. p. 223-39.

BAIRNER, A. y HWANG, D.J. (2011), "Representing Taiwan: international sport, ethnicity and national identity in the Republic of China", International Review for the Sociology of Sport, 46/3, p. 231-48.

BAKER, A.; BOYD, T. (eds.) (1997). Out of bonds: sports, media and the politics of identity. Bloomington: Indiana University Press.

BAKER, N. (1995). "The amateur ideal in a society of equality. Change and continuity in post-second world war British sport, 1945-48”. En: International Journal of the History of Sport, 12/1. p. 99-126.

BAKER, W.J. (1986). Jesse Owens: an american life. Nueva York: Free Press. (1992). "Muscular marxism and the Chicago counter-olympic games of 1932". En: International Journal of the History of Sport, 9. p. 397-410.

BILLIG, M. (1995). Banal nationalism. Londres: Sage.

BIMPER, A.Y. y HARRISON, L. (2011), "Meet at the crossroads: african american athletic and racial identity", Quest, 63/3, p. 275-88.

BLAIN, N.; BOYLE, R.; O'DONNELL, H. (1993). Sport and national identity in the European media. Leicester: Leicester University Press.

BLOXHAM, A. "The Queen in Ireland: symbolic visit to Croke Park". The Telegraph, 18.05.2011. 
BONDE, H. (2009). "Nationalism in the age of extremes: taking danish gymnastics to the world". En: International Journal of the History of Sport, 26/10. p. 1414-35.

BRÜGGEMEIER, F.-J. (2010). "Das Wunder von Bern: the 1954 football world cup, the german nation and popular histories". En PALETSCHEK, S. (ed.) (2010). Popular historiographies in the 19th and 20th centuries: cultural meanings, social practices. Oxford: Berghahn. p. 188-200.

BURCA, M. de (1999). The GAA: a history. Dublín: Gill and Macmillan.

BURKE-KENNEDY, E. "Croke Park event is hugely symbolic". Irish Times, 18.05.2011.

CARLSON, C. (2011). "The motherland, the godfather, and the birth of a basketball dynasty: american efforts to promote basketball in Lithuania". En: International Journal of the History of Sport, 28/11. p. 1479-95.

CASPISTEGUI, F.J. (2004). "“La resbaladiza arista de un monte erguida sobre dos abismos': mujer y deporte en España (1900-1950)". En: Memoria y Civilización, 7. p. $129-174$.

CASTILLO, J.C. (2007). "The concept of loyalty and the challenge of internationalisation in post-modern spanish football". En: International Journal of Iberian Studies, 20/1. p. 23-40.

CHO, Y. (2008). "Broadcasting major league baseball as a governmental instrument in South Korea". En: Journal of Sport \& Social Issues, 32/3. p. 240-54.

CINGIENE, V.; LASKIENE, S. (2004). "A revitalized dream: basketball and national identity in Lithuania". En: International Journal of the History of Sport, 21/5. p. $762-$ 79.

COAKLEY, J.; DUNNING, E. (eds.) (2000). Handbook of sport studies. Londres: Sage.

CRONIN, M. (1999). Sport and nationalism in Ireland: Gaelic games, soccer and Irish identity since 1884. Dublín: Four Courts Press.

MURPHY, W.; ROSE, P. (eds.) (2009). The Gaelic Athletic Association, 1884-2009. Dublín: Irish Academic Press.

CROSS, G. (2001). “Consumerism”. En: STEARNS, P.N. (ed.) (2001). V. p. 77-88.

DINE, P. (1997). "Peasants into sportsmen: modern games and the construction of french national identity". En DINE, P.; HENRY, I.P. (eds.) (1997). The symbolism of sport in France. Stirling: Stirling French Publications.

DONGGUANG, P. (2002). "The genesis of olympic matters in China (1895-1948)". En: Olympic Review, 27/45. p. 45-53.

DUNNING, E. (1994). "Sport as a male preserve: notes on the social sources of masculine identity and its transformations". En BIRELL, S.; COLE, C. (eds.) (1994), Women, sport and culture. Urbana: Human Kinetics. p. 163-79.

SHEARD, K. (2005 [1979]). Barbarians, gentlemen and players: a sociological study of the development of rugby football. Oxford: Martin Robertson.

DYRESON, M. (2008). “America's athletic missionaries': political performance, olympic spectacle and the quest for an american national culture, 1896-1912". En: International Journal of the History of Sport, 25/2. p. 185-203.

EDELMAN, R. (1993). Serious fun: a history of spectator sports in the USSR. Oxford: Oxford University Press.

(2002). "A small way of saying 'no': Moscow working men, Spartak soccer, and the Communist Party, 1900-1945”. En: American Historical Review, 107/5. p. 1441-74. 
EISEN, G.; WIGGINS, D.K. (eds.) (1994). Ethnicity and sport in North American history and culture. Westport: Greenwood Press.

ELIAS, N.; DUNNING, E. (1987). Quest for excitement. Sport and leisure in the civilising process. Oxford: Blackwell.

EVENSEN, B.J. (1996). When Dempsey fought Tunney: heroes, hokum, and storytelling in the Jazz Age. Knoxville: University of Tennessee Press.

FABRIZIO, F. (1976). Sport e fascismo. La politica sportiva del regime 1924-1936. Rimini: Guaraldi.

FAVERO, J.-P. (2008). "Italian immigration and sports in the iron-producing basin of Briey (1900-43)". En: International Journal of the History of Sport, 25/8. p. 974-92.

GARCÍA FERRANDO, M. (2005). "Mundialización y deporte. Paradojas de la glocalización”. En ARIÑO, A. (ed.) (2005). Las encrucijadas de la diversidad cultural. Madrid: CIS. p. 453-66.

GEBAUER, G. (1994). “Le nouveau nationalisme sportif'. En: Actes de la recherche en sciences sociales, 103. p. 104-7.

GEMS, G.R. (2006). The athletic crusade: sport and american cultural imperialism. Lincoln: University of Nebraska Press.

GIULIANOTTI, R. (1999). Football: a sociology of the global game. Cambridge: Polity Press.

GONZÁLEZ AJA, T. (ed.) (2002). Sporty autoritarismos. La utilización del deporte por el comunismo y el fascismo. Madrid: Alianza.

GORN, E. (1985). "The Manassa mauler and the fighting marine: an interpretation of the Dempsey-Tunney fights". En: Journal of American Studies, 19. p. 27-47.

GRUNEAU, R. (1988), "Modernization or hegemony: two views of sport and social development”. En HARVEY, J.; CANTELON, H. (eds.) (1988). Not just a game: essays in canadian sport sociology. Ottawa: University of Ottawa Press. p. 9-32.

GUOQI, X. (2008). Olympic dreams: China and sports, 1895-2008. Cambridge: Harvard University Press.

GUTTMANN, A. (1978). From ritual to record: the nature of modern sports. Nueva York: Columbia University Press.

(1991). Women's Sports: A History. Nueva York: Columbia University Press.

(2001). "Sports". En STEARNS, P.N. (ed.) (2001). V. p. 167-84.

THOMPSON, L.B. (2001). Japanese sports: a history. Honolulu: University of Hawaii Press.

HAND, D.; CROLEY, L. (2005). "Spanish identities in the European press: the case of football writing". En: International Journal of the History of Sport, 22/2. p. 298-313.

HARGREAVES, J. (1986). Sport, power and culture. Cambridge: Polity Press.

HARVEY, A. (2004). Beginnings of a commercial sporting culture in Britain, 17931850. Abingdon: Ashgate.

HEINRICH, A. (2003). "The 1954 soccer world cup and the Federal Republic of Germany's self-discovery". En: American Behavioral Scientist, 46/11. p. 1491-1505.

HESSE-LICHTENBERGER, U. (2003). Tor! The story of german football. Londres: WSC Books.

HOBERMAN, J. (1995). "Toward a theory of Olimpic Internationalism". Journal of Sport History, 22/1. p. 1-37. 
HOBSBAWM, E.J. (1993). "The Example of the English Middle Class". En KOCKA, J.; MITCHELL, A. (eds.) (1993). Bourgeois society in nineteenth-century Europe. Oxford: Berg.

RANGER, T. (eds.) (1996 [1983]). The invention of tradition. Cambridge: Cambridge University Press.

HOFMANN, A.R. (2008). "Between ethnic separation and assimilation: german immigrants and their athletic endeavours in their new American home country". En: International Journal of the History of Sport, 25/8. p. 993-1009.

HOLT, R. (1990). Sport and the working class in modern Britain. Manchester: Manchester University Press.

HONG, F.; HUA, T. (2002). "Sport in China: conflict between tradition and modernity, 1840 to 1930s". En MANGAN, J.A.; HONG, F. (eds.) (2002). Sport in Asian society past and present. Londres: Frank Cass. p. 189-212.

HONG, F.; MACKAY, D.; CRISTENSEN, K. (eds.) (2008). China gold: China's quest for global power and olympic glory. Great Barrington: Berkshire.

HORNE, J.; TOMLINSON, A.; WHANNEL, G. (1999). Understanding sport: an introduction to the sociological and cultural analysis of sport. Londres: Routledge.

HOULIHAN, B. (2000). "Politics and sport". En COAKLEY, J.; DUNNING, E. (eds.) (2000). p. 213-27.

HUNTER, J. (2003). "Flying the flag: identities, the nation, and sport". En: Identities, 10/4. p. 409-25.

JACKSON, S.J.; BATTY, R.; SCHERER, J. (2001). "Transnational sport marketing at the global/local nexus: the adidasification of the New Zealand All Blacks". En: International Journal of Sports Marketing \& Sponsorship, 3/2. p. 185-201.

JACKSON, S.J.; HOKOWHITU, B. (2002). "Sport, tribes, and technology: the New Zealand All Blacks Haka and the politics of identity". En: Journal of Sport and Social Issues, 26/2. p. 125-39.

JARVIE, G. (2003). "Internationalism and sport in the making of nations". En: Identities, 10/4. p. 537-51.

HWANG, D.-J.; BRENNAN, M. (2008). Sport, revolution and the Beijing Olympics. Oxford: Berg.

JARVIE, G.; WALKER, G. (eds.) (1994). Scottish sport in the making of the nation: ninety-minute patriots?. Leicester: Leicester University Press.

JINXIA, D. (2010). "The Beijing Games, national identity and modernization in China". En: International Journal of the History of Sport, 27/16-18. p. 2798-2820.

JOHNES, M. (2000). "Eighty minute patriots? National identity and sport in modern Wales". En: International Journal of the History of Sport, 17/4. p. 93-110.

JOHNSON, M.W. (2008). Studies in Central European histories: training socialist citizens: sports and the state in East Germany. Boston: Brill.

JONES, S.G. (1988). Sport, politics and the working class: organised labor and sport in interwar Britain. Manchester: Manchester University Press.

JORDAN, S. (2005). "Der deutsche Sieg bei der Weltmesiterschaft 1954: Mythos und Wunder oder historisches Ereignis?". En: Historical Social Research, 30/4. p. 263-81.

JUN, J.W. y LEE, H.M. (2012), "The globalization of sport and the mass-mediated identity of Hines Ward in South Korea", Journal of Sport Management, 26/2, p. 103-12. 
KING, C.R. (ed.) (2005). Native athletes in sport and society: a reader. Lincoln: University of Nebraska Press.

KRAUSS, W. (2003). "Football, nation and identity: german miracles in the postwar era”. En DYCK, N.; ARCHETTI, E.P. (eds.) (2003). Sport, dance and embodied identities. Oxford: Berg. p. 197-215.

KRUGER, A.; RIORDAN, J. (1996). The story of worker sport. Champaign: Human Kinetics.

KYLE, D.G. (1990). “E. Norman Gardiner and the decline of greek sport”. En KYLE, D.G.; STARK, G.D. (eds.) (1990). Essays in sport history and sport mythology. College Station: Texas A\&M University Press. p. 7-44.

LANFRANCHI, P. (2002). "Football, cosmopolitisme et nationalisme". En: Pouvoirs, 101/2. p. 15-25.

LEE, J.W.; BAIRNER, A. (2009). "The difficult dialogue: communism, nationalism, and political propaganda in North Korean sport". En: Journal of Sport and Social Issues, 33/4. p. 390-410.

LIN, C.-Y.; LEE, P.-C.; NAI, H.F. (2009). "Theorizing the role of sport in state-politics". En: International Journal of Sport and Exercise Science, 1/1. p. 23-32.

MACALOON, J.J. (1984). "Olympic games and the theory of spectacle in modern societies”. En MACALOON, J.J. (1984). Rite, drama, festival, spectacle: Rehearsals toward a theory of cultural performance. Philadelphia: Institute for the Study of Human Issues. p. 241-80.

MACRURY, I.; POYNTER, G. (2010). "“Team GB' and London 2012: the paradoxes of national and global identities". En: International Journal of the History of Sport, 27/16-18. p. 2958-75.

MAGUIRE, J. (1999). Global sport: identities, societies, civilizations. Cambridge: Polity Press.

(2006). "Sport and globalization: key issues, phases, and trends". En RANEY, A.A.; BRYANT, J. (eds.) (2006). Handbook of sports and media. Mahwah: Lawrence Erlbaum. p. 435-46.

MANDLE, W.F. (1987). The Gaelic Athletic Association and Irish nationalist politics, 1884-1924. Dublín: Gill and Macmillan.

MANGAN, J.A. (1985). The games ethic and imperialism: aspects of the diffusion of an ideal. Harmondsworth: Viking.

MARTIN, S. (2004). Football and Fascism: The National Game Under Mussolini. Oxford: Berg.

MCANALLEN, D.; HASSAN, D.; HEGARTY, R. (eds.) (2009). The evolution of GAA. Uladh, Éire agus Eile. Belfast: Ulster Historical Foundation.

MCGOVERN, P. (2002). "Globalization or internationalization? Foreign footballers in the English League". En: Sociology, 36/1. p. 23-42.

MCQUARRIE, F.A.E. (2010). "The struggle over worker leisure: an analysis of the history ot the workers' sports association in Canada". En: Canadian Journal of Administrative Sciences, 27/4. p. 391-402.

MEAD, C. (1985). Champion. Joe Louis, black hero in white America. Nueva York: Scribner.

MIGNON, P. (1999). "Le sport, facteur de paix ou de guerre à l'époque contemporaine”. En: Géopolitique, 66. p. 45-50. 
MILLER, T. et al. (2001). Globalization and sport: playing the world. Londres: Sage.

MILZA, P. (dir.) (2004). Le pouvoir des anneaux. Les jeux olympiques à la lumière de la politique, 1896-2004. París: Vuibert.

MITCHELL, S. (1977). "Women's participation in the Olympic Games, 1900-1926”. En: Journal of Sport History, 4. p. 208-28.

MORRIS, A.D. (1999). "'I can compete!' China in the Olympic Games, 1932 and 1936”. En: Journal of Sport History, 26. p. 545-66.

(2004). Marrow of the nation. A history of sport and physical culture in Republican China. Berkeley: University of California Press.

MROZEK, D. (1983). Sport and american mentality, 1880-1910. Knoxville: University of Tennessee Press.

NAISON, M. (1979). "Lefties and righties: the Communist Party and sport during the Great Depression". En: Radical America, 13. p. 47-59.

NIEHAUS, A. (2006). "'If you want to cry, cry on the green mats of Kôdôkan': expressions of japanese cultural and national identity in the movement to include judo into the Olympic programme". En: International Journal of the History of Sport, 23/7. p. 1173-92.

(2010). "Modernization and identity creation (1868-1920): the evolution of competitive swimming in Japan”. En: International Journal of the History of Sport, 27/3. p. 505-22.

NIELSEN, N.K. (2005). Body, sport and society in Norden countries: essays in cultural history. Aarhus: Aarhus University Press.

O'DONNELL, H. (1994). "Mapping the mythical: a geopolitics of national sporting stereotypes". En: Discourse and Society, 5. p. 345-80.

O'MAHONY, M. (2006). Sport in the USSR. Physical culture, visual culture. Londres: Reaktion Books.

OTOMO, R. (2007). "Narratives, the body and the 1964 Tokyo Olympics". En: Asian Studies Review, 31/2. p. 117-32.

POCIELLO, C. (1999). Sports et sciences sociales. Histoire, sociologie et prospective. París: Vigot.

POLLEY, M. (1998). Moving the goalposts. A history of sport and society since 1945. Londres: Routledge.

(2008). "History and sport". En HOULIHAN, B. (ed.) (2008). Sport and society: a student introduction. Londres: Sage. p. 56-74.

POPE, S.W. (1993). "Negotiating the 'folk highway' of the nation: sport, public culture and american identity, 1870-1940". En: Journal of Social History, 27/2. p. 327-40.

(1997). Patriotic games: sporting traditions in the American imagination, 18761926. Nueva York: Oxford University Press.

RADER, B.G. (1977). "The quest for subcommunities and the rise of american sport". En: American Quarterly, 29/4. p. 355-69.

(1983). "Compensatory sport heroes: Ruth, Grange and Dempsey". En: The Journal of Popular Culture, 16/4. p. 11-22.

REID, I.; JARVIE, G. (2000). "Sport, nationalisms and their futures". En JONES, R.; ARMOUR, K. (eds.) (2000). Sociology of sport. Theory and practice. Essex: Longman. p. 83-96. 
RIORDAN, J. (1977). Sport in Soviet society: development of sport and physical education in Russia and the USSR. Cambridge: Cambridge University Press.

(1991). Sport, politics and communism. Manchester: Manchester University Press.

SÁNCHEZ GARCÍA, R.; MALCOLM, D. (2010). "Decivilizing, civilizing or informalizing? The international development of mixed martial arts". En: International Review for the Sociology of Sport, 45/1. p. 39-58.

SAURE, F. (2009). "Beautiful bodies, exercising warriors and original peoples: sports, greek antiquity and national identity from Winckelmann to "Turnvater Jahn"'. En: German History, 27/3. p. 358-73.

SENN, A.E. (1988). "American Lithuanians and the politics of basketball in Lithuania, 1935-1939”. En: Journal of Baltic Studies, XIX/2. p. 146-56.

SHEARD, K.G. (1997). "Aspects of boxing in the western "civilizing process"". En: International Review for the Sociology of Sport, 32/1. p. 31-57.

SHUN, I. (1998). "The invention of martial arts. Kanô Jogorô and Kôdôkan Jûdô". En VLASTOS, S. (ed.) (1998). Mirror of modernity. Invented traditions of modern Japan. Berkeley: University of California Press. p. 163-73.

SILK, M.L.; ANDREWS, D.L.; COLE, C.L. (eds.) (2004). Sport and corporate nationalisms. Oxford: Berg.

SKINNER, J.; ZAKUS, D.; EDWARDS, A. (2008). "Coming in from the margins: ethnicity, community support, and the rebranding of the Australian soccer". Soccer and Society, 9/3. p. 394-404.

SMITH, A.; PORTER, D. (eds.) (2004). Sport and national identity in the post-war world. Londres: Routledge.

STEARNS, P.N. (ed.) (2001). Encyclopedia of european social history. From 1350 to 2000. Detroit: Charles Scribner's Sons.

STEINBERG, D.A. (1978). “The workers' sport internationals 1920-28”. En: Journal of Contemporary History, 13. p. 233-51.

STRUNA, N.L. (2001). "Reframing the direction of change in the history of sport". En: International Journal of the History of Sport, 18/4. p. 1-15.

SUGDEN, J.; BAIRNER, A. (1986). "Northern Ireland: sport in a divided society". En: ALLISON, L. (ed.) (1986). The politics of sport. Manchester: Manchester University Press. 90-117.

(1993). Sport, sectarianism and society in a divided Ireland. Leicester: Leicester University Press.

SUNDEEN, J.T. (2001). “A 'kid's game'?: little league baseball and national identity in Taiwan”. En: Journal of Sport and Social Issues, 25/3. p. 251-65.

TAGSOLD, C. (2002). Die Inszenierung der kulturellen Identität in Japan. Das Beispiel der Olympischen Spiele Tôkyô 1964. Munich: Iudicium.

URBINA GAITÁN, C. (2001). Costa Rica y el deporte 1873-1921. Un estudio acerca del origen del fútbol y la construcción de un deporte nacional. Heredia: Euna.

- (2006). "Fútbol e identidad nacional en Centroamérica. Un análisis comparativo de los casos de Guatemala, El Salvador y Costa Rica". En: Revista de Ciencias Sociales, 113-114. p. 177-87.

WALTON, J.K. (2001). "Policing leisure”. En STEARNS, P.N. (ed.) (2001). V. p. 15565. 
WHEELER, R. (1978). “Organized sport and organized labor: the workers' sports movement". En: Journal of Contemporary History, 13. p. 191-210.

ZHOUXIANG, L. (2011). "Sport, nationalism and the building of the modern chinese nation state (1912-49)". En: International Journal of the History of Sport, 28/7. p. 1030-54.

HONG, F. (2010). "From celestial empire to nation state: sport and the origins of chinese nationalism (1840-1927)". En: International Journal of the History of Sport, 27/3. p. 479-504.

\section{Notas}

1 El video con el que se explicó la historia de la GAA, señalaba: "for over a century, gaelic games have remained the keenest expression of our Irishness" (The Journal, 18.05.2011). Todavía a comienzos de los años ochenta los grupos de castigo del Sinn Fein utilizaban un stick de hurling en sus represalias (Sugden, Bairner, 1986: 98).

2 Irish Examiner, 16.05.2011; Burke-Kennedy, 2011; de los nueve condados del Ulster, sólo uno estuvo representado (Belfast Telegraph, 18.05.2011; The Independent, 18.05.2011).

3 "GAA President welcomes Queen to Croke Park", www.breakingnews.ie [visto el 10.08.2011].

4 Un reflejo de ello fue la reglamentación del boxeo y de otras modalidades de lucha iniciadas por las élites pero adoptadas por los excluidos (Sheard, 1997), aunque se habla ya de un proceso des-civilizador (Sánchez García, Malcolm, 2010). Elias, Dunning, 1987.

5 Durante el siglo XIX, en los países nórdicos, "[f]arsighted politicians and leaders undestood that body culture was a shor cut to creating both diligent and nationcommited citizens. So, the state had a keen interest in constructing national(istic) leisure by means of body culture" (Nielsen, 2005: 48). Valga como dato la asistencia a las finales de la copa de fútbol inglesa (1872, 2.000; 1885, 10.000; 1901, 101.000; 1923, 200.000. Cross, 2001: 80). En el Reino Unido se produjo una expansión del deporte comercializado desde fines del XVIII (Harvey, 2004: 7-30).

6 Lin, Lee, Nai, 2009. En Francia pronto se asumió la centralidad institucional en la promoción deportiva, en parte por sus posibilidades nacionalizadoras (Dine, 1997).

7 Un punto de partida es Coakley, Dunning, 2000. La sociología del deporte, más antigua, criticó a la historia su escasa conceptualización (Horne, Tomlinson, Whannel, 1999: 73-94). Desde la historia, Polley, 2008: 56-74. Una visión global en Pociello, 1999.

8 Avanzados los ochenta se estudió la relación entre historia del deporte y nación -aunque hay ejemplos previos-, con hitos como Hargreaves, 1986; y Sugden, Bairner, 1986. Parte del rechazo al estudio de este vínculo, se debió a la visión del deporte como ajeno a la política, pese a los manifiestos ejemplos en sentido contrario. 
La imagen del deporte aficionado, caballeresco, al margen de enfrentamientos partidistas, se ha mantenido con fuerza. Hunter, 2003.

9 Guttmann (1978: 54) indicaba siete características de la fase moderna: secularismo, igualdad, especialización, racionalización, burocracia, cuantificación y marcas. Una visión crítica desde la teoría de la hegemonía, Gruneau, 1988. Un modelo alternativo: Struna, 2001. Otro hito en la división en dos: Dunning, Sheard, 2005 [1979]: 1-16.

10 Para Bairner (2009), la nación tiene sustancia material más allá de su visión como comunidad imaginada o inventada, e insiste en los paisajes como defensa del primordialismo y en la idea de que el deporte, incluso en nuestra época, mantiene elementos de atracción más allá de lo monetario.

11 Billig, 1995: 120, 122; O’Donnell, 1994. Es revelador el uso por Coca Cola de la canción “Wawin' flag” de K'naan, en el mundial de Sudáfrica 2010.

12 Dyreson, 2008; Mrozek, 1983. Para la tesis de la hegemonía cultural, Pope, 1993 y 1997.

13 Morris, 2004: 185-229. Algo similar ocurrió en Japón, que inicialmente incorporó el modelo occidental, pero la reacción nacionalista de fines del XIX recuperó y adaptó sus propias tradiciones, como la natación (Niehaus, 2010).

14 Para estos combates, Gorn, 1985; Evensen, 1996. El resumen de alguno de ellos en www.youtube.com/watch? $v=-$ OeeCfbahwQ. Dempsey como encarnación de un modelo compensatorio en Rader, 1983. Muy similares son boxeadores argentinos como Firpo, Gatica o Monzón (Archetti, 2001: 97-111).

15 Baker, 1986; Mead, 1985; Bimper y Harrison, 2011, entre otros; King, 2005: 22-39, 79-103. Más problemas tuvieron los indígenas en Centroamérica, excluidos incluso de la integración a través del deporte (Urbina Gaitán, 2001 y 2006).

16 Alamillo, 2010; Hofmann, 2008. El deporte entre los inmigrantes en EE.UU. y su capacidad para generar asociacionismo en: Rader, 1977; Eisen, Wiggins, 1994. Un ejemplo europeo: Favero, 2008; y uno australiano: Skinner, Zakus, Edwards, 2008.

17 Un ejemplo contrario es el de la adscripción fascista (por su cercanía al modelo japonés y nazi, y el rechazo al soviético) de la gimnasia danesa de Niels Bukh en el período de entreguerras (Bonde, 2009).

18 Edelman, 2002. Sobre el deporte en la URSS: Riordan, 1977; Edelman, 1993; O’Mahony, 2006. Para la RDA: Johnson, 2008.

19 Baker, 1995. Destaca la imagen de integración y superación de desigualdades asociada a la sede londinense de las olimpiadas de 2012, promocionada como actitud universal (Macrury, Poynter, 2010).

20 Shun, 1998; Niehaus, 2006. Es interesante por su reflexión sobre el impacto transformador de los juegos de 1964, también en lo identitario: Otomo, 2007. Guttman, Thompson, 2001.

21 Algunos ejemplos: la utilización del beisbol juvenil por Taiwan para reafirmar su presencia internacional frente a China y como instrumento nacionalizador (Sundeen, 
2001; o, más general, Bairner y Hwang, 2011). Frente a él, los juegos olímpicos de Pekín y la modernización y consolidación de China como potencia mundial (Jinxia, 2010). El vínculo entre medios de comunicación y deporte con una finalidad nacionalizadora en Corea del Sur (Cho, 2008; Jun, Lee, 2012). Por último, Túnez y el deporte a principios del siglo XXI, "[i]] est à la fois un instrument de propagande pour le régime politique, mais aussi un lieu d'expression de la nouvelle symbolique identitaire qu'il veut incarner" (Abbassi, 2007: 141-2).

22 ¿Sirvió la utilización de la haka del equipo de rugby de Nueva Zelanda en la publicidad de Adidas como instrumento de refuerzo identitario o más bien debilitó un potente elemento simbólico? Jackson, Batty, Scherer, 2001; Jackson, Hokowhitu (2002: 127) afirman que "transnational investment in the national sport provides privileged access to the past, present, and future meaning of not only the game but also the nation". El anuncio, de 1999, en www.youtube.com/watch? $v=$ JuiGF4TGI9w\&feature $=$ fvsr.

23 Jarvie, Walker, 1994. Otros casos de este patriotismo de ocasión, en el fútbol inglés: Abell, 2007; en Gales: Johnes, 2000; o la dificultad unificadora del deporte en espacios muy politizados como Escocia o Irlanda (Bairner, 1996).

24 Bairner, 2001: 176; García Ferrando, 2005. Para Grant Jarvie, la relación deportenacionalismo está garantizada (Jarvie, 2003: 543). Véase: Reid; Jarvie, 2000.

25 Para Jarvie (2003: 547 y 548) internacionalización se refiere "to the extension of activities across national boundaries"; globalización a la extensión geográfica, pero sobre todo "to the functional integration of such internationally dispersed activities"; y cosmopolitanismo se asocia "with a range of identities rather than with one national team defined in only territorial terms". Véase Mcgovern, 2002.

26 Giulianotti, 1999. Lo aplica a España Castillo, 2007. Allen Guttman (2001) es reacio y mantiene el esquema pre-moderno/moderno.

\section{El autor}

Francisco Javier Capístegui es profesor Agregado de la Universidad de Navarra y ha estudiado diferentes aspectos relacionados con el deporte en obras como Guerras danzadas. Fútbol e identidades locales y regionales en Europa (2001 -con J.K. Walton-); Cien años de relación entre los navarros y el deporte (1901-2001) (2010 - con S. Leoné-). También ha investigado sobre el carlismo (El naufragio de las ortodoxias (1997); Aventuras de un gentleman en la tercera carlistada (2007 -con P. Larraz y J. Ansorena-); Una historia por descubrir. Materiales para el estudio del carlismo (2010)) y diversas cuestiones metodológicas en obras como La nueva historia cultural (1996 -con I. Olábarri-) o The strength of history at the doors of the new millennium (2005 -con I. Olábarri-) 